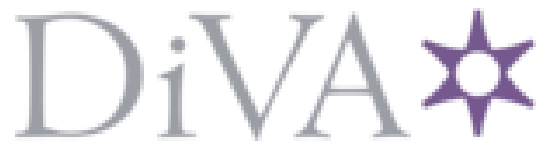

http://www.diva-portal.org

Preprint

This is the submitted version of a paper published in Journal of Cognition and Culture.

Citation for the original published paper (version of record):

Eriksson, K., Coultas, J C., De Barra, M. (2016)

Cross-Cultural Differences in Emotional Selection on Transmission of Information

Journal of Cognition and Culture, 16(1-2): 122-143

https://doi.org/10.1163/15685373-12342171

Access to the published version may require subscription.

N.B. When citing this work, cite the original published paper.

Permanent link to this version:

http://urn.kb.se/resolve?urn=urn:nbn:se:mdh:diva-31794 


\title{
Cross-cultural differences in emotional selection on transmission
}

\section{of information}

\author{
Kimmo Eriksson ${ }^{1,2}$ \\ Julie C. Coultas 1,3 \\ Mícheál de Barra ${ }^{1}$
}

${ }^{1}$ Centre for the Study of Cultural Evolution, Stockholm University, Stockholm, Sweden

${ }^{2}$ School of Education, Communication and Culture, Mälardalen University, Västerås, Sweden

${ }^{3}$ University of Sussex, Brighton, UK

Word count (excl. abstract and references): 6,545

Acknowledgments: This work was supported by the Swedish Research Council [grant number 20092390].

Correspondence should be addressed to Kimmo Eriksson, Centre for the Study of Cultural Evolution, Stockholm University, Wallenberglab., SE-10691 Stockholm, Sweden (e-mail: kimmo.eriksson@mdh.se). 


\title{
Cross-cultural differences in emotional selection on transmission of information
}

\begin{abstract}
Research on cultural transmission among Americans has established a bias for transmitting stories that have disgusting elements (such as exposure to rats and maggots). Conceived of as a cultural evolutionary force, this phenomenon has been termed emotional selection. In a series of online studies with Americans and Indians we investigate whether there are cultural differences in emotional selection, such that the transmission process favours different kinds of content in different countries. The first study found a bias for disgusting content (rats and maggots) among Americans but not among Indians. Four subsequent studies focused on how country interacts with kind of emotional content (disgusting vs. happy surprises and good news) in reactions to transmission of stories or information. Whereas Indian participants, compared to Americans, tended to be less interested in, and excited by, transmission of stories and news involving common disgust-elicitors (like rats), the opposite pattern held for transmission of happy surprises and good news (e.g., the opening of a new public facility). We discuss various possible explanations and implications.
\end{abstract}




\section{Introduction}

Social life revolves around people telling each other things, such as anecdotes, rumours, gossip, information, and advice. Some of it will be their own original ideas and observations, but often people retell what they have previously heard from another source. In such repeated cultural transmission of the same material, any systematic bias in what type of content tends to be passed along will result in an amplification of that kind of content within the culture. In particular, only a small set of stories or rumours will eventually reach a large share of the population and any bias in the transmission process should make this most successful material stand out in some way. From an adaptive point of view, we might expect a bias for information that is likely to maximise fitness, for instance by being important and accurate. In analogy with natural selection in biological evolution, Heath, Bell and Sternberg (2001) suggested that the working of such a bias might be called "informational selection" in cultural evolution. However, the case of urban legends shows that success in cultural transmission does not necessarily rely on importance or accuracy. Heath et al. proposed that the success of urban legends might instead be explained by “emotional selection". Narrowing their focus to the emotion of disgust, they conducted studies that provided two different kinds of evidence of emotional selection. First, analysis of a pool of successful urban legends showed clear evidence of over-representation of story elements known to elicit disgust (e.g., rats and cockroaches, mutilations, and disgusting food). Second, when stories were manipulated with respect to level of disgust, participants in experiments tended to be more willing to pass along stories the more disgusting they were.

Although the findings of Heath et al. (2001) are thought-provoking and have been cited many times, their cross-cultural validity is an open question. The pool of urban legends was compiled from mainly American sources, and the experiments used only American college students as participants. A later study replicated disgust-based emotional selection using both laboratory and online experiments, still with American samples (Eriksson \& Coultas, in press). We know of no previous attempts at replication outside Western culture. Indeed, given the general impact of the paper of Heath et al. (2001), the empirical work that has actually followed-up on the concept of emotional selection is surprisingly limited. Below we describe the three most relevant studies, all of which used American or Australian college students as participants. 
In a field study of college students who visited a hospital morgue, Harber and Cohen (2005) followed how the students shared this emotional experience with friends, and how these stories were retold and then retold again in secondary and tertiary sharings. Primary, secondary and tertiary sharings were all predicted by the level of the primary sharer's emotional reaction. Whereas urban legends are essentially fictional stories, this finding extends the scope of emotional selection on repeated transmission to include actual experiences of someone relatively close in the narrator's social network.

Peters, Kashima and Clark (2009) extended the lab studies of Heath et al. (2001) by manipulating a range of emotions in social anecdotes. In addition to replicating the original finding of increased willingness to pass along an anecdote that elicits higher levels of disgust, they found a similar effect of higher levels of happiness, interest, or surprise. Some other emotions (sadness, fear, and contempt) seemed to have less effect. Berger (2011) suggested that the differences in communicability between emotions may be partially explained by the associated physiological arousal. In experiments he found higher willingness to share some neutral material with a friend in conditions that created higher arousal, whether from watching film clips evoking high-arousal emotions such as anxiety or amusement (vs. low-arousal emotions such as sadness or contentment) or just from jogging lightly in place (rather than sitting still).

\section{Reasons to expect cross-cultural differences in emotional selection on transmission}

It is not necessarily the case that emotional selection biases are culturally universal. Indeed, cross-cultural differences have been predicted. Specifically, Schaller (2006, p. 100) predicted that disgust-based emotional selection of stories "would be especially strong under conditions in which parasite prevalence, and thus the potential risk of contagion, was especially high." The rationale for this prediction is the role of the disgust system as a disease avoidance mechanism, i.e., an adaptation that influences avoidance behaviour in order to minimise the risk of disease (Oaten et al., 2009; Curtis, 2011; Davey, 2011). To the extent that disgust-relevant information supports adaptive avoidance behaviour it would serve a population if individuals were prone to transmit such information, and particularly so in environments where disgust-elicitors represent an especially high risk of contagion.

However, the more recent work of Peters et al. (2009) and Berger (2011) provides basis for an alternative prediction. As described earlier, the findings in these papers indicate that emotional selection on 
transmission is not specific to disgust but rather associated with high-arousal emotions more generally.

This generality of emotional selection makes an adaptationist explanation that is specific for the disgustbased case less plausible. Instead, the arousal theory of emotional selection suggests another possibility: that high exposure to disgust elicitors may decrease their power as triggers of cultural transmission, if by habituation their salience as disgust cues is reduced (Rozin 2008). In the words of Oaten et al. (2009, p. 308), "frequent exposure to corpses, sick individuals, refuse, open sewers, and poor-quality food and hygiene may act to reduce disgust sensitivity toward the very type of stimuli for which one would want to maintain maximal sensitivity."

So far we have discussed biologically oriented hypotheses, whereby cross-cultural differences in emotional selection would arise because of differences in the environment. A complementary possibility is that emotional selection is moderated by cultural factors, such as taboos and norms about appropriate topics of conversation. Indeed, within our own cultures, there are clearly contexts when disgust-related discussions are acceptable and other contexts where the same subjects would be considered "improper". It is plausible that there are across-culture as well as within-culture differences in the acceptability of disgust-related discussion.

\section{The case of United States vs. India}

A comprehensive understanding of cross-cultural differences in emotional selection would require experiments on cultural transmission in a wide range of countries. Eriksson and Coultas (in press) conducted most of their studies online, using the Amazon Mechanical Turk (AMT) to recruit American participants. The AMT does not enable the kind of large data collection in many countries that would be ideal, because its pool of users is entirely dominated by individuals from India and United States (Eriksson and Simpson, 2010). What AMT does provide is the possibility of comparative studies between these two countries, which is a good first step. When we searched the internet for Indian urban legends we ended up with the impression that there was nothing corresponding to the American abundance of disgusting urban legends, and that popular stories in India more often focused on some moral aspect of behaviour. Indeed, there are several reasons why these two particular countries are interesting to compare with respect to 
emotional selection of the kind studied by Heath et al. (2001), i.e., a bias for transmission of stories about rats, cockroaches, mutilations and disgusting food.

For one thing, pathogens are much more prevalent in India than in United States (Fincher et al., 2008; Murray and Schaller, 2010). Thus, Schaller's prediction would in this case predict even higher willingness to transmit disgust-relevant information among Indians than among Americans.

On the other hand, many of the disgusting elements in the urban legends studied by Heath et al. (2001) were animals such as rats and cockroaches, and cross-cultural research has found that such disgustrelevant animals tend to evoke less negative emotion in Indians than in Americans (Davey, McDonald, Hirisave, Prabhu et al., 1998). This finding is consistent with the habituation hypothesis, as many Indian cities lack adequate waste disposal or sanitation infrastructure (WHO / UNICEF, 2010) and Indian participants are likely to encounter associated disgust stimuli more frequently. Thus we hypothesise that there will be robust cross-cultural differences in the emotional response to disgusting story elements such as rats and cockroaches, with less emotional intensity and arousal among Indians than Americans. Because these emotional responses are important triggers of social transmission (Berger, 2011; Peters et al., 2009; Rimé, 2009), we also expect corresponding cross-cultural differences in the power of these stimuli to trigger social transmission

Finally, there are stark differences between India and United States in terms of religious and moral systems. The Hindu Indian moral system places particular emphasis on the moral foundation of purity and divinity (Rozin, 1988; Rozin, Lowery, Imada and Haidt, 1999; Shweder, Haidt, Horton and Joseph 2008). It seems plausible that, compared to other cultures, individuals in such a moral system may experience higher levels of emotional response to such stimuli that are regarded as impure and lower levels to stimuli that the purity/sanctity system is not concerned with. To quote Rozin (1999, p. 26), "in the United States, disgust focuses on food, animal nature, and interpersonal elicitors, whereas in Hindu India, it has evolved further from its origins, with central foci in the interpersonal and moral domains."

In summary, there are several theoretical reasons why India and United States may differ in emotional selection of disgusting stories about things like rats, cockroaches, mutilations and disgusting food. On balance, we should probably expect less transmission of this kind of disgusting stories among Indians than 
Americans. However, we do not expect emotional selection to be generally weaker among Indians.

Following Peters et al. (2009) we therefore decided to study transmission based on other emotions as well.

\section{Overview of studies}

Below we present five studies of cultural transmission of stories and information. All studies were conducted online with Indian and American users of the Amazon Mechanical Turk. The sampled populations of AMT users differed somewhat between India and United States in terms of gender and age. However, we found gender and age to have little or no importance for cultural transmission. For simplicity, we therefore report results of country comparisons without controlling for these variables.

Study 1 compared Indians and Americans on their willingness to pass along, as well as their recall of, stories manipulated to be either high or low on disgust. Study 2 compared willingness to pass along six different emotions. The results motivated a focus on disgust and surprise in the remaining studies. Study 3 compared arousal levels from reading either disgusting or surprising story themes. Study 4 compared willingness to pass along, and association of emotions with, information in two domains: elicitors of disgust and positive surprises. Study 5 replicated the previous study and included measures of interest in the information and change in arousal from reading about surprises after reading about disgust elicitors.

\section{Study 1: Recall and pass along of low and high disgust stories}

Eriksson and Coultas (in press) manipulated urban legends to be either highly disgusting or non-

disgusting. In an American sample they found that disgust versions tended to be rated as more likely to be passed along, replicating one of the findings of Heath et al. (2001). They also found disgust versions of stories to be better recalled, consistent with a large body of evidence for enhanced recall of emotionally charged events (Kensinger and Schacter, 2008). Here we investigate whether these effects of disgusting content differ between Americans and Indians. 


\section{Method}

\section{Participants}

Fifty American AMT users (56\% female, mean age 35 years) and 50 Indian AMT users (36\% female, mean age 30 years) completed an online experiment.

\section{Materials and design}

We used a between subjects design with two conditions, disgust and non-disgust. Both samples had about half of participants in each condition. Depending on condition, participants read different versions of an urban legend-type story in two parts about a traveller named Jasmine; the same manipulation was previously used by Eriksson and Coultas (in press, Experiment 1). First Jasmine eats a pizza and finds an object stuck in her teeth; depending on the version, this object is either a rat's tooth (disgust) or an olive stone (non-disgust). Next Jasmine bakes a cake and then realizes that the cake is either baked with maggot infested flour (disgust) or has turned out really delicious (non-disgust).

After reading the story respondents rated the likelihood that they would pass it along, using a 7-point Likert-type scale from $1=$ not at all likely to pass along the story to $7=$ very likely to pass along the story. They also rated how disgusting they found the story, on a scale from $1=$ not at all disgusting to $7=$ very disgusting. On a separate screen respondents were then asked to recall the story as accurately as they could.

\section{Coding}

The story consisted of 14 sentences (seven in each part), which served as the units of recall in the coding stage. Two coders (one author and one person who was unaware of the purpose of the study) went through the stories and marked the items that were recalled accurately. A sentence was judged to be recalled if the basic content was present, i.e., the recall did not need to be verbatim (Lyons and Kashima, 2003; Eriksson and Coultas, in press). For each participant, this yielded a recall score between 0 and 14 . Inter-rater reliability was very high $(\alpha=.99)$. In the analysis below we used the average scores of the two raters. 


\section{Results}

Disgust ratings. As a manipulation check, we conducted a two-by-two ANOVA of the effects of condition and country upon disgust ratings. There was a main effect of condition, $F(1,96)=116.36, p<.001$, with disgust ratings higher in the disgust condition $(M=5.35)$ than in the non-disgust condition $(M=1.88)$.

There was no main effect of country, $F(1,96)=0.44, p=.51$. However, there was an interaction between condition and country, $F(1,96)=3.98, p=.049$, such that the difference between conditions was higher among Americans $(M=5.56$ vs. 1.44$)$ than among Indians $(M=5.13$ vs. 2.30$)$. This interaction suggests that Indians were less sensitive to the presence or absence of rats and maggots in the stories.

Pass along ratings. Next we conducted a two-by-two ANOVA of the effects of condition and country upon pass along ratings. There was a main effect of condition, $F(1,96)=5.33, p<.023$, with pass along ratings higher in the disgust condition $(M=4.08)$ than in the non-disgust condition $(M=3.33)$. There was a main effect of country as well, $F(1,96)=17.97, p<.001$, with pass along ratings higher among Indians $(M=$ 4.44) than among Americans $(M=2.94)$. Thus Indians generally reported higher interest in passing along these short stories. However, there was an interaction between condition and country, $F(1,96)=6.57, p=$ .012 , such that the difference between conditions was present only among Americans ( $M=3.80$ vs. 2.08) and not among Indians ( $M=4.39$ vs. 4.48). In other words, Americans-but not Indians— showed a bias for stories with rats and maggots, i.e., the kind of emotional selection previously demonstrated in studies using only American participants (Heath et al., 2001; Eriksson \& Coultas, in press).

Consistent with these findings, correlational analysis of the two ratings across both conditions show that disgust ratings predicted pass along ratings among Americans $(r=.52, p<.001)$ but not among Indians $(r$ $=-.02, p=.91)$.

Recall. Finally we conducted a two-by-two ANOVA of the effects of condition and country upon recall scores. There were no significant main effects, $p \mathrm{~s}>.28$, but there was an interaction between condition and country, $F(1,96)=6.26, p=.014$; whereas Americans showed a tendency for better recall in the disgust condition than in the non-disgust condition $(M=9.02$ vs. 8.22$)$, Indians showed worse recall in the disgust condition ( $M=7.13$ vs.9.15). 
Across countries and conditions, recall scores and pass along ratings were positively correlated $(r=.22, p$ $=.031)$. This is consistent with both measures partially reflecting the emotional impact.

\section{Discussion}

In this study, we found American and Indian respondents react differently to disgusting vs. non-disgusting versions of a story. While Americans tended to be more likely to pass along, and better at recalling, the disgusting than the non-disgusting version, replicating previous work (Heath et al., 2001; Eriksson and Coultas, in press), the opposite pattern was shown by Indians. These results indicate that there are crosscultural differences in emotional selection of stories. Specifically, inclusion of common disgust-elicitors like rats and maggots seem to increase cultural transmission among Americans but not among Indians.

\section{Study 2: Pass along ratings for experiences of different emotions}

Eriksson and Coultas (in press, Experiment 4) asked AMT users in the United States how likely they would be to pass along stories conveying either of six emotions. Here we replicate this study with both American and Indian respondents to investigate whether there are any group differences in individuals' stated preferences for transmission of emotional content.

\section{Participants}

One hundred and fifty nine American AMT users (58\% female, mean age 37 years) and 156 Indian AMT users (38\% female, mean age 29 years) completed an online survey.

\section{Materials and design}

The instructions read: "This set of six questions is about how likely you would be to pass along certain stories. Imagine that someone told you a story about a person you don't know. The story describes how this person experiences something. Given the type of experience, you may be more or less likely to pass along the story (retell it to someone else). Please rate how likely you would be to pass along the story for each of the following types of content." Then followed six types of content, each of which was described as "A story where the person experienced something that made him feel X", where $\mathrm{X}$ was one of the six emotions anger, amusement, sadness, fear, disgust, and surprise (counter-balanced between different 
versions). For each item, ratings were made on a Likert scale from $1=$ not at all likely to pass along the story to $5=$ very likely to pass along the story.

\section{Results}

A two-by-six mixed ANOVA of the effects of country and emotion upon pass along ratings revealed no significant main effect of country, $F(1,312)=3.13, p=.08$, but a significant main effect of emotion, $F(5,312)=53.70, p<.001$, and an interaction between country and emotion, $F(5,312)=12.55, p<.001$. The country difference in the profile of pass along ratings for different emotions is illustrated in Figure 1. Analysis of simple effects using t-tests revealed that, compared to American respondents, Indians were less willing to transmit stories where a person experienced anger, $p=.001, d=0.36$, or disgust, $p=.003$, $d=0.32$, but more willing to transmit stories about fear, $p<.001, d=0.45$, and surprise, $p<.001, d=0.54$. There were no significant country differences with respect to stories about amusement or sadness.

\section{Discussion}

The American data closely replicated the pattern found by Eriksson and Coultas (in press): In ratings of the likelihood to pass along stories about six different emotions, amusement came out highest and sadness lowest. In the Indian data, in contrast, surprise came out highest and disgust was lowest. Indeed, whereas transmission of disgust stories was lower among Indians than Americans, the opposite was true for surprise stories. Study 2 is, of course, limited in that the emotions were just mentioned and no actual story content was presented, but at least it gives tentative support for the interpretation that the lower emotional selection among Indians found in Study 1 is specific to disgust and does not represent a generally lower interest in transmitting emotional stories. Our remaining studies will test this conclusion further, focusing on the two emotions of surprise and disgust.

\section{Study 3: Arousal from disgusting and surprising story themes}

Berger (2011) found that sharing of emotional content is linked to the level of arousal of the potential sharer. Here we follow up on Study 2 by investigating whether the patterns of arousal levels from reading either disgusting or surprising story themes are different between Indians and Americans. 


\section{Participants}

One hundred and nineteen American AMT users (53\% female, mean age 36 years) and 145 Indian AMT users (39\% female, mean age 31 years) completed an online experiment.

\section{Materials and design}

Participants were randomly assigned to one of two conditions: disgust or surprise. In the disgust condition, participants were asked to imagine that an acquaintance told them a story about a person who "ate something infested with worms; trod deeply in buman feces; drank from a bottle in which floated a rat; slept in a bed that crawled with cockroaches; was sprayed in the face with urine; or something else along the same lines." In the surprise condition, the manipulation instead read "realized there was something unexpected in bis wallet; saw something unusual in the street; found a group of people had organized something without the person suspecting anything; learnt that a famous brand name had suddenly changed; experienced something he had not even considered possible to happen; or sometbing else along the same lines." Both conditions then continued as follows: "Imagine that the story was told with enough detail so that it would be easy for listeners to share the emotion of the person in the story. With this in mind, please read the above list again!"

As a manipulation check we first asked which of four basic emotions (anger, disgust, fear, surprise) best described the emotional content of these stories. Following Berger (2011), arousal was then measured on three 7-point scales (passive-active, mellow-fired up, and low-high energy), averaged to form an arousal index $(\alpha=.68)$.

\section{Results}

One hundred and twelve US respondents and 94 Indians correctly identified the emotion. Because an incorrect answer suggests lack of either attention or comprehension, only those who correctly identified the emotion were kept for the following analysis (leaving 108 participants in the surprise condition and $\mathrm{N}=98$ in the disgust condition).

We conducted a two-by-two ANOVA of the effects of condition and country upon arousal. There was a main effect of condition, $F(1,202)=11.60, p<.001$, with arousal higher in the surprise condition $(M=$ 
4.41) than in the disgust condition $(M=3.96)$. There was no main effect of country, $F(1,202)=0.67, p=$ .41. However, there was an interaction between condition and country, $F(1,202)=11.87, p<.001$. Analysis of simple effects revealed that Indians reported much higher arousal in the surprise condition than in the disgust condition $(M=4.61$ vs. 3.60), $p<.001, d=0.87$. In contrast, Americans reported the same arousal in both conditions $(M=4.22$ vs. 4.23$), p=.98$.

\section{Discussion}

The results followed the pattern expected from the findings in the previous two studies: reading disgusting (vs. surprising) story themes yielded relatively lower arousal in Indians than in Americans. This indicates that there are cross-cultural differences in the emotional impact of different story themes. In particular, reading about common disgust-elicitors like rats and cockroaches seem to have less impact on Indians than on Americans.

\section{Study 4: Passing along of non-social news, either disgusting or good}

The studies above, as well as related experimental research on the topic of emotional selection, have focused on legends and anecdotes of a social nature: some individual experiences something (Heath et al., 2001; Peters et al., 2009; Eriksson \& Coultas, in press). However, for the argument behind Schaller's (2006) prediction of a greater bias for transmitting disgusting stories under conditions of high pathogen prevalence, it is the informational rather than the social aspect that is really relevant. For this reason, we now shift focus to transmission of non-social information. As a non-social correspondence to stories about people experiencing, say, rats and bugs (i.e., the disgust themes in Study 3), we study news of rats and bugs in the local environment. Let us call this disgusting local news. Similarly, corresponding to stories about people finding or seeing unexpected positive things (i.e.,the surprise themes in Study 3), we study news about unexpected positive changes in the local environment. Let us call this good local news. Following the previous studies, we want to compare transmission of disgusting local news and good local news among Indians and Americans. 


\section{Participants}

One hundred and ninety one American AMT users (52\% female, mean age 34 years) and 188 Indian AMT users (37\% female, mean age 30 years) completed an online survey.

\section{Materials and design}

Participants were presented with a list of possible pieces of information "that a friendly neighbor might pass along to you when you meet in the street." They were asked to estimate the likelihood that they would pass the same piece of information along "if you were to meet another friendly neighbor shortly thereafter." Likelihood estimates were given on a percent scale from 0 to 100.

The list included four pieces of disgusting local news (there are rats in the street in the next block; there are bed bugs in the local hotel; someone has vomited under the bench at the bus stop; a man on the bus took out his glass eye) and three pieces of good local news (the park is having a new lawn laid; the local charity received a large donation yesterday; the new road has opened already).

Finally, to test that these items are indeed perceived as disgusting news and good news, respectively, we presented a list of six emotions (happy, amused, sad, angry, disgusted, fearful) and asked participants for each item to tick any of these emotions hearing this piece of news would evoke in them.

\section{Results}

Factor analysis of pass along items. The scree plot of a principal components analysis of the seven pass along likelihoods supported a two-factor solution, explaining $56 \%$ of the variance. The results of a varimax rotation yielded one factor with the four disgusting news items: vomit (factor loading .79), glass eye (.76), bed bugs (.63), and rats (.50), forming a pass along index for disgusting news $(\alpha=.63)$; and another factor with the three good news items: new lawn (.87), new road (.78), and donation (.68), forming a pass along index for good news $(\alpha=.71)$.

Associated emotions. Consistent with being perceived as good news, on average $58 \%$ of Americans and $62 \%$ of Indians thought good news items would evoke happiness; the second most common emotion was amusement (14\% and 20\%, respectively). Similarly, on average $76 \%$ of Americans and $45 \%$ of Indians 
thought disgust news items would evoke disgust; the second most common emotion was fearful $(17 \%$ in both samples).

Effects of country and kind of news on pass along. A two-by-two mixed ANOVA of the effects of country and kind of news upon pass along likelihood revealed no significant main effect of country, $F(1,377)=3.23$, $p$ $=.07$, but a significant main effect of kind of news, $F(1,377)=100.22, p<.001$, with good local news more likely to be passed along than disgusting local news ( $M=55.13$ vs. 41.96). This effect was qualified by an interaction between country and kind of news, $F(1,377)=36.76, p<.001$, such that the likelihood of passing along disgusting news was lower in India than in United States $(M=36.24$ vs. 47.59$), p<.001$, $d=0.54$, whereas the opposite tended to hold for good news $(M=57.48$ vs. 52.81$), p=.058, d=0.19$.

\section{Discussion}

Here we replicated the pattern of the previous studies also for non-social news about the local environment. Compared to American participants, Indians indicated less willingness to pass along disgusting local news, whereas their willingness to pass along good news was somewhat higher instead. We conclude that the cross-cultural difference in transmission of disgusting stories does not seem to be based in the social aspect (e.g., in the capacity to identify with the person experiencing some disgusting event). Rather, it seems to be the disgust-elicitors themselves (e.g., rats, bugs, vomit and glass eyes) that elicit different responses in India and United States.

\section{Study 5: Change in affect from hearing non-social news}

Cultural transmission entails both receiving and passing along (Eriksson \& Coultas, in press). To obtain a more reliable measure of how likely a piece of information is to be transmitted we complemented the pass along questions in Study 4 by asking also how likely the participant would be to ask to hear more on each topic from the informant. We also tested whether reading the two kinds of local news items produces different changes in the reader's current affect among Indians and Americans. The two-dimensional "affect grid" was used, providing a convenient way of repeatedly measuring self-reported arousal as well as intensity and valence of feelings (Russell, Weiss, \& Mendelsohn, 1989). From previous work we expect 
transmission to be facilitated by higher arousal and of higher intensity of feelings, independent of valence (Berger, 2011; Rimé, 2009).

\section{Participants}

One hundred and ninety nine American AMT users (40\% female, mean age 35 years) and 194 Indian AMT users (36\% female, mean age 30 years) completed an online experiment.

\section{Materials and design}

To begin with, participants were introduced to usage of the "affect grid" (Russell et al., 1989). This is a 9by-9 grid where the respondent selects one cell to indicate their current state of affect with respect to two dimensions: arousal (from extreme sleepiness to extreme arousal) and valence (from extremely unpleasant feelings to extremely pleasant feelings). Participants were then presented with the local news items from Study 4 and reported their mood on the affect grid. Mood was reported at two different times during the experiment: once after reading (and estimating transmission likelihoods for) the disgusting news, and once after the good news. To average out any order effects, half the participants started with the disgusting news items and the other half started with the good news items. For each news item, participants estimated two likelihoods: that they would ask to hear more on the topic and that they would pass the same piece of information along (the latter measure same as in Study 4).

\section{Results}

Arousal and valence of emotions after reading disgusting local news or good local news. The two affect grid tasks yielded two measures of arousal, one after reading each kind of news. We conducted a two-by-two mixed ANOVA of the effects of country and kind of news upon arousal. No main effects were significant, but there was the expected interaction between country and kind of news, $F(1,392)=4.41, p=.036$, such that arousal after good news was higher in India than in United States ( $M=5.63$ vs. 5.22$), p=.013, d=0.25$, whereas arousal after disgusting news exhibited no country difference $(M=5.41$ vs. 5.31$), p=.54$.

We then similarly analysed the measures of valence. There was a main effect of kind of news, $F(1,392)=$ 133.95, $p<.001$, with more pleasant feelings after good news than after disgusting news, and also a main effect of country, $F(1,392)=11.28, p<.001$, with Indians reporting more pleasant feelings than 
Americans, both after good news ( $M=6.89$ vs. 6.47$), p=.018, d=0.24$, and after disgusting news $(M=$ 5.73 vs. 5.06$), p=.002, d=0.31$. Indeed, there was no significant interaction between country and kind of news.

Factor analysis of transmission items. The scree plot of a principal components analysis of the total of 14 likelihoods supported a two-factor solution, explaining $51 \%$ of the variance. The results of a varimax rotation yielded one factor of four pass along likelihoods and four hear more likelihoods for the four disgusting news items (loadings between .62 and .72), forming a transmission index for disgusting news $(\alpha$ $=.82$ ); and a second factor with pass along likelihoods and hear more likelihoods for the three good news items (loadings between .73 and .82), forming a transmission index for good news ( $\alpha=.86$ ).

Effects of country and kind of news on transmission. We conducted a two-by-two mixed ANOVA of the effects of country and kind of news upon transmission. There was a main effect of country, $F(1,392)=11.85, p$ $<.001$, with higher transmission among Indians than Americans; there was also a main effect of kind of news, $F(1,392)=166.51, p<.001$, with higher transmission of good news than disgusting news. As expected there was also an interaction between country and kind of news, $F(1,392)=38.25, p<.001$. Simple effects analysis revealed that transmission of good news was higher in India than United States $(M$ $=64.41$ vs. 50.54), $p<.001, d=0.62$, whereas transmission of disgusting news if anything showed the opposite pattern $(M=38.01$ vs. 41.24$), p=.11, d=0.16$.

Effects of arousal and valence of feelings upon transmission of different kinds of news. Finally, we investigated if individuals' tendency to transmit a given kind of news was predicted by their self-reported affect after reading each kind of news. For illustrative purposes we categorised arousal as either low (values 1-3), medium (4-6), or high (7-9). Valence of feelings was similarly categorised as negative, neutral, or positive. Figure 2 shows, separately for Indians and Americans, how transmission of good news and disgusting varied with arousal and valence. First note that, in both countries, higher arousal was associated with higher transmission of both kinds of news (left panels of Figure 2). This was expected from previous work on the relationship between arousal and transmission (Berger, 2011). Because the two arousal measures were highly inter-correlated, we controlled for arousal after disgusting news when computing the partial correlation between transmission of and arousal after good news $(r=.19, p=.009$, in India; $r=.16, p=$ 
.026, in United States). Analogously, we controlled for arousal after good news when computing the partial correlation between transmission of and arousal after disgusting news $(r=.16, p=.025$, in India; $r$ $=.16, p=.022$, in United States).

Second, in both countries, neutral feelings after reading good news were associated with lower transmission than more intense feelings, independent of valence (top right panel of Figure 2). Again, this was expected from previous work on how more intense emotions trigger sharing (Rimé, 2009). The partial correlation between transmission of good news and intensity (disregarding valence) of feelings after good news, controlling for intensity of feelings after disgusting news, was indeed positive in both countries $(r=$ $.27, p<.001$, in India; $r=.25, p<.001$, in United States).

For disgusting news, however, another pattern emerged (bottom right panel of Figure 2). Specifically, Indians who felt negative feelings after reading disgusting news were less likely to engage in transmission, whereas Americans exhibited no relation at all between valence of feelings and transmission of disgusting news. An ANOVA confirmed the interaction between country and valence category, $F(2,388)=5.32, p=$ .005 .

\section{Discussion}

Several points are worthy of discussion. First, by inclusion of questions about how likely participants would be to ask to hear more about various pieces of news, this study extended the pass along scale of Study 4 to a more reliable transmission scale for the same two kinds of local news (good news and disgusting news). Using this measure we again replicated the same basic interaction as in the previous studies: Compared to Americans, Indians were relatively less interested in transmission of disgusting news (vs. good news). We also found the corresponding interaction for arousal, replicating the finding of Study 3 using another design.

Higher arousal tends to be associated with higher transmission (Berger, 2011; Rimé, 2009). Indeed, we found a positive relationship between arousal and transmission for both kinds of news and in both countries. Thus, part of the explanation for the country level difference in transmission of disgusting 
content vs. surprises/good news may be that culture moderates the arousal evoked by different kinds of content.

However, differences in arousal seem not be the entire story. That something else is going on too was indicated by our analysis of the effect of pleasant and unpleasant feelings on transmission. Indians, but not Americans, tended to be reluctant to engage in transmission of disgusting news if they felt unpleasant feelings. Importantly, this phenomenon was specific to news with disgusting content and did not show up for good news. Perhaps culture defines the appropriateness of transmitting different kinds of content that leaves you with unpleasant feelings.

\section{General discussion}

Heath et al. (2001) found that Americans are more likely to pass on urban legends the more disgusting elements the story contains. The aim of the present work was to investigate whether this bias for disgusting content is a cultural universal or whether it varies between countries. We conducted five online experiments with Indians and Americans, using various kinds of materials (urban legends, labels of emotions experienced in social anecdotes, descriptions of story themes, and local news), and various measures of transmission and emotional impact. Compared to Americans, we found Indians to be consistently less likely to transmit stories and news about common disgust-elicitors like rats and bugs, but more likely to transmit positive surprises and good news.

In the introduction we outlined various reasons to expect this kind of cross-cultural difference. In brief, we expected a country difference in the emotional impact of the abovementioned common disgustelicitors. For reasons both environmental (higher exposure to these disgust-elicitors) and cultural (focus of disgust shifted toward moral and interpersonal domains), the average emotional impact was expected to be smaller in India than in United States (Davey et al., 1998; Oaten et al., 2009; Rozin, 1999). We would then expect a corresponding difference in transmission of this kind of content, because emotional intensity and arousal trigger transmission (Berger, 2011; Rimé, 2009). On the whole our findings are consistent with this account. However, Study 5 indicated the existence of another mechanism as well: Indians who experienced unpleasant feelings after reading news about some common disgust-elicitors (such as rats, bugs, vomit) were particularly unlikely to engage in transmission of such news. This 
phenomenon was not present among Americans, nor did Indians exhibit it in transmission of another kind of news. Some kind of cultural norm may be acting on transmission of emotional content. More detailed research into the interplay between emotions, cultures, and transmission is clearly warranted.

To summarise, we have conducted a first attempt at measuring cross-cultural differences in emotional selection on cultural transmission. We focused on Indians and Americans sampled from an internet site (Amazon Mechanical Turk). Given the finding of robust differences between these countries, several extensions suggest themselves. For instance, the exposure hypothesis would predict geographical variability in transmission patterns also within countries. Another issue is to explain why happy surprises and good news seem to make more impact on Indians than Americans. A third research direction could be to examine whether Indian and American socially transmitted culture actually differs in ways predicted by the emotional selection theory. In other words, are stories about, say, rats really less popular in the Indian pool of stories than in the American counterpart? Finally, the question of general principles underlying cross-cultural differences in transmission of stories and news should ultimately be addressed in studies involving participants from many more than just a pair of countries.

\section{Funding}

This work was supported by the Swedish Research Council [grant number 2009-2390].

\section{References}

Berger, J. (2011). Arousal increases social transmission of information. Psychological Science, 22, 891 - 893.

Curtis (2011). Why disgust matters. Philosophical Transactions of the Royal Society B. 366, 3478- 3490.

Davey, G. C. L. (2011). Disgust the disease-avoidance emotion and its dysfunctions. Philosophical Transactions of the Royal Society B. 366, 3453- 3465.

Davey, G. C. L., Mc Donald, A. S., Hirisave, U., Prabhu, G. G., Iwawaki, S., Im Jim, C., Merckelbach, H., de Jong, P. J., Leung, P. W. L. \& Reimann, B. C. (1998). A cross-cultural study of animal fears. Behaviour Research and Therapy, 36, 735-750. 
Eriksson, K. and Coultas, J. C. (in press). Corpses, maggots, poodles and rats: Emotional selection operating in three phases of cultural transmission of urban legends. Journal of Cognition and Culture.

Eriksson, K., and Simpson, B. (2010). Emotional reactions to losing explain gender differences in entering a risky lottery. Judgment and Decision Making, 5, 159-163.

Fincher, Thornhill, Murray and Schaller, (2008). Pathogen prevalence predicts human cross-cultural variability in individualism/collectivism. Proceedings of the Royal Society. B, 275, 1279-1285.

Harber, K. D., \& Cohen, D. J. (2005). The emotional broadcaster theory of social sharing. Journal of Language and Social Psychology, 24, 382-400

Heath, C., Bell, C. and Sternberg, E. (2001). Emotional selection in memes: The case of urban legends. Journal of Personality and Social Psychology, 81, 6, 1028-1041.

Kensinger, E.A. and Schacter, D.L. (2008). Memory and Emotion. In M. Lewis, J.M. Haviland-Jones \& L. Feldman Barrett (Eds.), Handbook of Emotions, third edition, (pp. 601-617), New York: Guilford Press.

Lyons, A. and Kashima, Y. (2003). How are stereotypes maintained through communication? Journal of Personality and Social Psychology, 85, 989-1005.

Murray, D. R. and Schaller, M. (2010). Historical prevalence of infectious diseases within 230 geopolitical regions: A tool for investigating origins of culture. Journal of Cross-Cultural Psychology, 41, 99 -108

Oaten, M., Stevenson, R. J. \& Case, T. I. (2009). Disgust as a disease-avoidance mechanism. Psychological Bulletin. Vol. 135, No. 2, 303-321.

Olantunji, B. O., Moretz, M. W., McKay, D., Bjorklund, F., de Jong, P. J., Haidt, J., Hursti, T. J., Imada, S., Koller, S., Mancini, F., Page, A. C. \& Schienle, A. (2009). Confirming the three-factor structure of the Disgust Scale-Revised in eight countries. Journal of Cross-Cultural Psychology, 40, 234-255.

Peters, K., Kashima, Y. and Clark, A. (2009). Talking about others: Emotionality and the dissemination of social information. European Journal of Social Psychology, 39, 207-222. 
Rimé, B. (2009). Emotion elicits the social sharing of emotion: theory and empirical review. Emotion Review, 1, 60-85.

Rozin, P. (1999). Food is fundamental, fun, frightening, and far-reaching. Social Research, 66, 9-30.

Rozin, P. (2008). Hedonic "adaptation": Specific habituation to disgust/death elicitors as a result of dissecting a cadaver. Judgment and Decision Making, 3, 191-194.

Rozin, P., Lowery, L., Imada, S. and Haidt, J. (1999). The CAD triad hypothesis: A mapping between three moral emotions (contempt, anger, disgust) and three moral codes (community, autonomy, divinity). Journal of Personality and Social Psychology, 76, 574-586.

Russell, J. A., Weiss, A., \& Mendelsohn, G. A. (1989). Affect grid: A single-item scale of pleasure and arousal. Journal of Personality and Social Psychology, 57, 493-502.

Schaller, M. (2006). Parasites, behavioral defenses, and the social psychological mechanisms through which cultures are evoked. Psychological Inquiry, 17, 96-137.

Shweder, R. A., Haidt, J., Horton, R. and Joseph, C. (2008). The cultural psychology of the emotions: Ancient and renewed. In M. Lewis, J. Haviland-Jones and L. Feldman-Barrett (eds.). Handbook of Emotions, third edition (pp. 409-427). New York: Guilford.

WHO / UNICEF (2010) Progress on sanitation and drinking-water - 2010 update. Geneva: World Health Organization. 
Figure 1. Mean pass along ratings in India and United States for each of six emotions. (Bars indicate standard errors.)

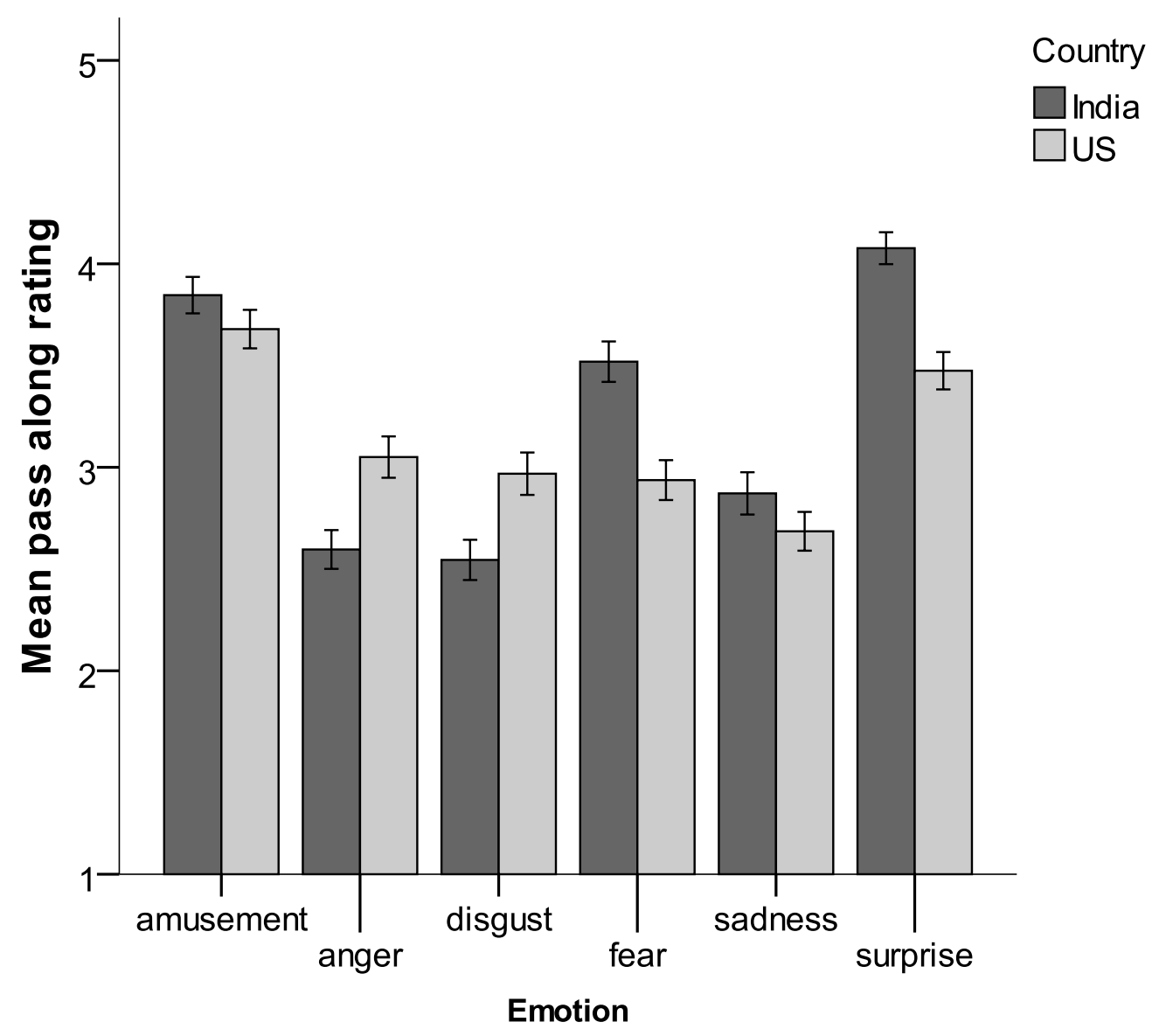


Figure 2. Transmission of good news (top) and disgusting news (bottom) in United States and India, depending on self-reported arousal (left) and valence of feelings (right) after reading the news.

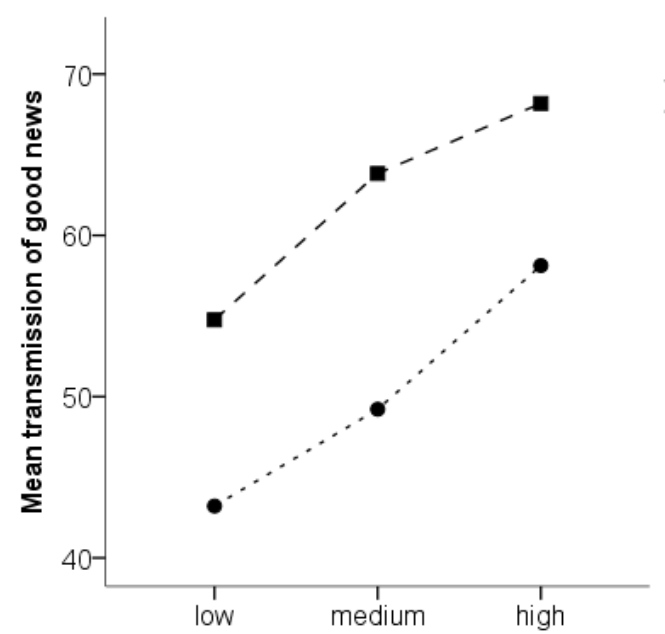

Arousal after reading good news

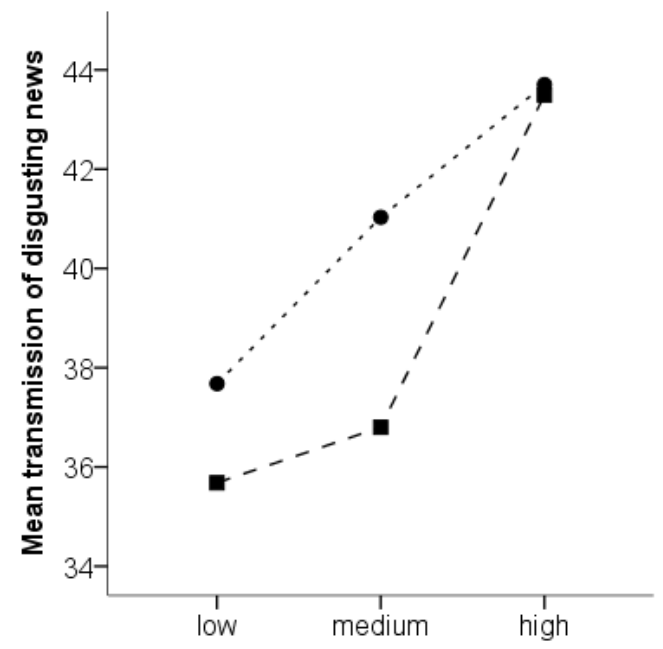

Arousal after reading disgusting news
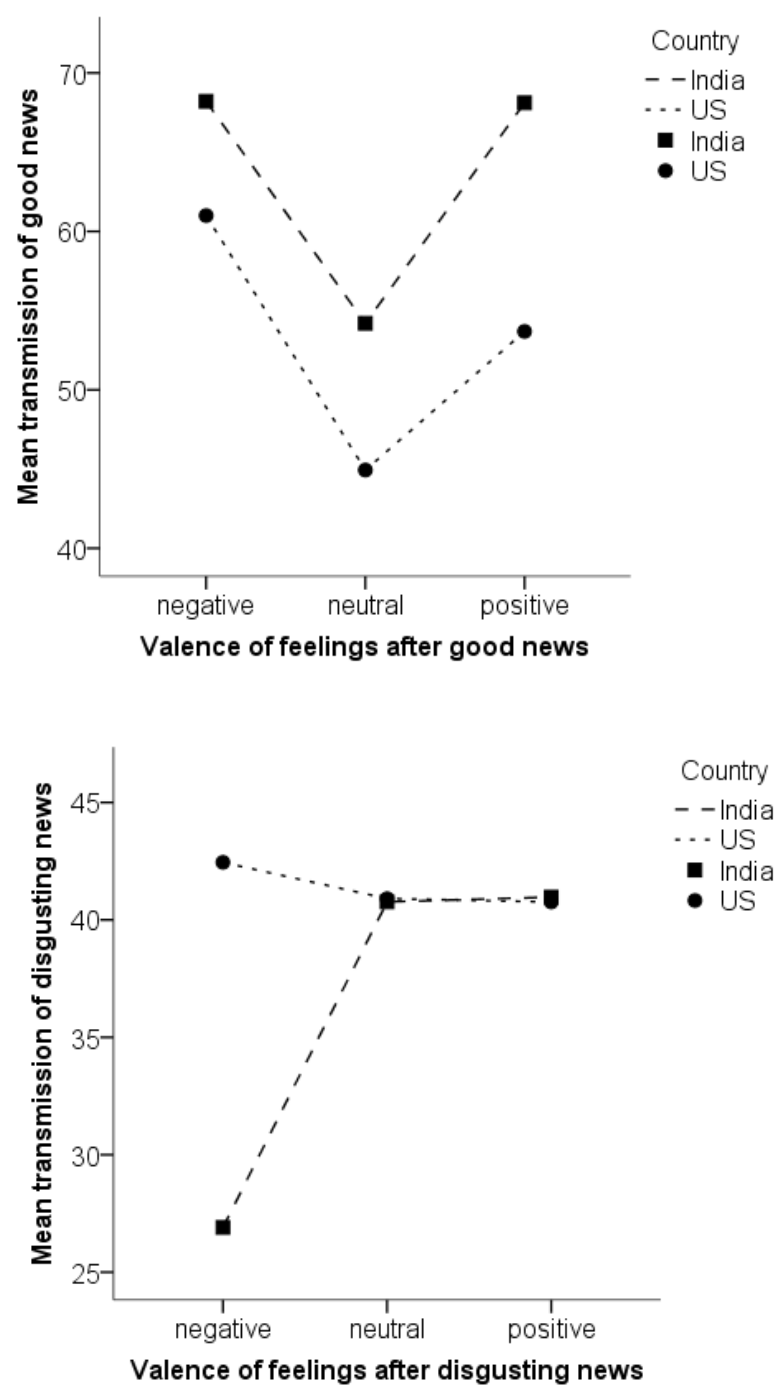